\title{
Caracterización de lesiones en columna vertebral mediante tomografía computarizada en canes de Lima, Perú
}

\author{
SPINE INJURIES CHARACTERIZATION BY COMPUTED TOMOGRAPHY IN CANINES \\ in Lima, Peru \\ Patricia Shimose C. ${ }^{1,2}$, Eben Salinas $C_{.}{ }^{1,3}$
}

\section{Resumen}

El objetivo del presente estudio fue caracterizar, mediante tomografía computarizada, lesiones en columna vertebral en canes. Los diagnósticos tomográficos fueron relacionados con las características demográficas de cada can en estudio (sexo, tamaño, raza y edad). Se evaluaron 79 diagnósticos tomográficos realizados entre junio de 2010 y julio de 2014. Los resultados indicaron mayor frecuencia de lesiones en canes machos de raza definida, tamaño mediano y en el estrato etario de 4-7 años. Se obtuvieron 22 diagnósticos tomográficos, siendo los más frecuentes la hernia discal medial $(32.8 \%, 26 / 79)$ y la calcificación laminar del saco dural $(11.4 \%, 9 / 79)$. Se identificaron 115 lesiones en columna vertebral, siendo más afectada la región lumbar (48.7\%, 56/115). A nivel de los espacios intervertebrales, la región torácica T12-T13 presentó el mayor número de lesiones $(14 \%, 16 / 115)$, especialmente discopatías.

Palabras clave: tomografía; discopatías; condrodistroficas; hernia discal

\section{Abstract}

The objective of this study was to characterize spine injuries in dogs by computed tomography. The tomographic diagnoses were related to the demographic characteristics of dogs under study (sex, size, breed, and age). A total of 79 tomographic diagnoses were evaluated between June 2010 and July 2014. The results showed a greater frequency of lesions in males of defined breed, medium size and in the age group of 4-7 years. Twentytwo tomographic diagnoses were obtained, the most frequent being medial disc herniation $(32.8 \%, 26 / 79)$ and laminar calcification of the dural sac $(11.4 \%, 9 / 79)$. A total of 115

Carrera de Medicina Veterinaria y Zootecnia, Universidad Cientifica del Sur, Lima, Perú

${ }^{2}$ E-mail: pshimoseciudad@gmail.com

${ }^{3}$ E-mail:esalinasc@cientifica.edu.pe

Recibido: 18 de julio de 2017

Aceptado para publicación: 7 de diciembre de 201 
lesions were identified in the spine, the lower spine being more affected $(48.7 \%, 56 / 115)$. At the level of the intervertebral spaces, the thoracic region T12-T13 presented the largest number of lesions $(14 \%, 16 / 115)$, especially discopathies.

Key words: tomography; discopathies; chondrodystrophic; disc herniation

\section{INTRODUCCIÓN}

La lesión de la médula espinal o mielopatía es la complicación más frecuente producto de la injuria de la columna vertebral o de la degeneración del disco intervertebral (McKee, 2000). En los caninos, las discopatías intervertebrales suelen ser la causa principal de las lesiones que afectan la médula espinal, conllevando a la presentación de signos neurológicos. Las discopatías se desarrollan principalmente en regiones límites de la columna vertebral como la cérvico torácica o toraco lumbar, por ser regiones de mayor estrés vertebral (Simpson, 1992; Lim et al., 2010).

La enfermedad degenerativa del disco intervertebral involucra un cambio en la estructura del núcleo pulposo del disco, generalmente por deshidratación, lo que desencadenará en una extrusión (hernia Hansen tipo 1) o protrusión (hernia Hansen tipo II) de este núcleo (Downes et al., 2009). La invasión del material herniado producirá un trauma directo sobre la medula espinal, raíces nerviosas y nervios espinales. Este evento desencadenará los signos neurológicos, según su gravedad, como el dolor, ataxia, paresia y pérdida de la sensación del dolor. Se ha establecido la alta predisposición en la hernia tipo 1 en canes jóvenes (2-4 años) y de características condrodistroficas como en el Dachshund (Bray y Burbidge, 1998). Otras causas de lesiones vertebrales y medulares son las alteraciones lumbosacras, fracturas, enfermedades neoplásicas y disco espondilitis; sin embargo, estas causas suelen presentarse en poca frecuencia en los canes al compararse con las discopatías.
La presentación clínica de las lesiones dependerá de la zona afectada, del tiempo que transcurrió desde que se originó la patología y del tipo de lesión causada. En lesiones hiperagudas, la presentación de los signos es súbita y pueden progresar en poco tiempo del dolor a la parálisis completa de los miembros inervados por la región de la columna afectada. Además, deben considerarse las repercusiones sistémicas del trauma inicial como las lesiones cerebrales o trauma torácico y abdominal (Bali et al., 2009). Si existe una compresión medular aguda (hernia Hansen tipo 1), los pacientes presentarán signos típicos como la cifosis de la columna vertebral producto del dolor a ese nivel, grados de ataxia, paresia o parálisis en miembros anteriores o posteriores, según la región afectada de la columna. Los signos que se presentarán en lesiones crónicas o degenerativas como las hernias de disco tipo 2 o tumores serán progresivos y generalmente relacionados a hiperestesia en la región afectada, ataxia y paresia, pero rara vez se presentará parálisis o afectaciones súbitas (Lecouteur y Grandy, 2002). La pérdida de la movilidad y la percepción del dolor, así como la incontinencia urinaria son factores determinantes para calificar una lesión medular según su gravedad y determinar el pronóstico (Coates, 2004).

Por lo tanto, es importante realizar el examen neurológico en base a la anamnesis y la exploración especializada de los pacientes. El objetivo inicial debe ser el lograr la neurolocalización de la lesión de acuerdo con los signos identificados y la historia del paciente. El siguiente paso será el planteamiento de los diagnósticos diferenciales, para finalmente identificar la etiología mediante herramientas diagnosticas por imágenes dispo- 
nibles (Pellegrino, 2014). Las radiografías simples de columna vertebral han sido la herramienta inicial que aproximan al diagnóstico; sin embargo, el diagnóstico definitivo necesita de medios de diagnóstico imagenológico más sensibles como las mielografías, tomografías o resonancias magnéticas (Coates, 2000; Widmer y Thrall, 2013).

La radiografía simple y la mielografía, debido a su limitada resolución de contraste, solo aportan evidencia indirecta de los cambios patológicos de la médula espinal (Olby et al., 2003). La resonancia magnética es la técnica imagenológica más precisa para evaluar patologías de tejidos blandos como la médula espinal, pero su alto costo y tiempo prolongado de exposición del paciente para adquirir las imágenes limita su uso (Dennis, 2003). Es por ello que la tomografía computarizada (TC) es el método más utilizado pues permite establecer una imagen directa de los cambios patológicos, pudiendo hacer una diferenciación superior de los tejidos blandos y evitar la superposición de estructuras subyacentes (Olby et al., 2000; Axlund y Hudson, 2003); además, la toma de las imágenes requiere cortos periodos de tiempo, evitando tiempos prolongados de anestesia en el paciente politraumatizado (Hecht et al., 2009).

El objetivo de este estudio fue caracterizar las lesiones en columna vertebral en canes diagnosticados mediante TC en Lima Metropolitana.

\section{Materiales y Métodos}

El estudio comprendió el total de historias clínicas e informes tomográficos de columna vertebral, obtenidos entre junio de 2010 y julio de 2014 en clínicas veterinarias de Lima Metropolitana, Perú. Se recolectaron datos sobre características demográficas (sexo, estrato etario, raza y tamaño), descripción, tipo y ubicación de la lesión (región anatómica de la columna vertebral) y diagnóstico tomográfico. Todas las tomografías de este estudio se realizaron en un mismo centro de diagnóstico y fueron obtenidas a través de un tomógrafo de segunda generación Siemens dual (de dos cortes), modelo Somaton Spirit +.

Para las variables de tipo cualitativo se determinó la proporción de cada resultado en sus respectivos estratos y se resumieron en tablas de frecuencias. Los datos fueron expresados en porcentajes.

\section{Resultados}

Se evaluaron 79 informes y sus respectivas historias clínicas de caninos correspondientes a diagnósticos tomográficos de columna vertebral. El 59.5\% fueron machos y la edad más frecuente de presentación correspondió al estrato etario de 4-7 años (38\%), la mayoría de casos correspondieron a razas definidas $(72.2 \%)$ y el tamaño más frecuente fue el mediano (50.6\%) (Cuadro 1).

Cuadro 1. Características demográficas de 79 pacientes caninos con tomografía computarizada de columna vertebral

\begin{tabular}{lcc}
\hline $\begin{array}{l}\text { Características } \\
\text { demográficas }\end{array}$ & $\mathrm{n}$ & $\%$ \\
\hline Sexo & 47 & 59.5 \\
$\quad$ Macho & 32 & 40.5 \\
$\quad$ Hembra & & \\
Estrato etario (años) & 14 & 17.7 \\
$\quad 0.5-3$ & 30 & 38.0 \\
$4-7$ & 28 & 35.4 \\
$\quad 8-11$ & 7 & 8.9 \\
12-16 & & \\
Raza & 59 & 72.2 \\
$\quad$ Definida & 20 & 27.8 \\
$\quad$ No definida & & \\
Tamaño & 19 & 24.1 \\
$\quad$ Miniatura y pequeño & 40 & 50.6 \\
$\quad$ Mediano & 20 & 25.3 \\
$\quad$ Grande &
\end{tabular}


Cuadro 2. Distribución de los diagnósticos tomográficos más frecuentes de lesiones vertebrales en caninos, según las características demográficas más frecuentes

\begin{tabular}{|c|c|c|c|c|c|}
\hline \multirow[b]{2}{*}{ Diagnóstico tomográfico } & \multicolumn{2}{|c|}{ Ocurrencia } & \multicolumn{3}{|c|}{$\begin{array}{l}\text { Característica demográfica más } \\
\text { frecuente }\end{array}$} \\
\hline & $\mathrm{n}$ & $\%$ & $\begin{array}{l}\text { Grupos } \\
\text { etarios } \\
\text { (años) }\end{array}$ & Tamaño & Raza \\
\hline Hernia discal medial & 26 & 32,8 & $8-11$ & Mediano & Beagle \\
\hline $\begin{array}{l}\text { Calcificación laminar del saco } \\
\text { dural }\end{array}$ & 9 & 11.4 & $8-11$ & Pequeño & $\begin{array}{l}\text { Dachshund } \\
\text { / Shih Tzu }\end{array}$ \\
\hline Hernia discal paramedial izquierda & 8 & 10.1 & $4-7$ & Mediano & Beagle \\
\hline Protrusión discal & 6 & 7.5 & $8-11$ & Grande & Labrador \\
\hline Hernia discal paramedial derecha & 5 & 6.3 & $4-7$ & Mediano & Cocker \\
\hline $\begin{array}{l}\text { Hernia discal medial y paramedial } \\
\text { izquierda }\end{array}$ & 4 & 5.1 & $12-16$ & Pequeño & Shih Tzu \\
\hline Otros $^{1}$ & 21 & 26.8 & & & \\
\hline Total & 79 & 100.0 & & & \\
\hline
\end{tabular}

1 Neoplasia vertebral, luxofractura, fusión intervertebral, estenosis foraminal bilateral, absceso vertebral, fractura de cuerpo vertebral, espondiloartrosis, enfisema medular, sindesmofitos, estenosis foraminal derecha, calcificación del ligamento amarillo, calcificación del ligamento longitudinal anterior, calcificación del ligamento longitudinal posterior, calcificación laminar del cordón medular, hernia discal medial y paramedial derecha, hernia discal paramedial izquierda y derecha

Cuadro 3. Regiones de columna vertebral y espacios intervertebrales afectados en 79 canes evaluados por tomografía computarizada ( $\mathrm{n}=115$ lesiones)

\begin{tabular}{lccc}
\hline $\begin{array}{l}\text { Región de columna } \\
\text { vertebral }\end{array}$ & Espacio intervertebral & Número & \multirow{2}{*}{ Total } \\
\hline \multirow{3}{*}{ Cervical } & C4-C5 & 5 & \multirow{2}{*}{17.4} \\
& C6-C7 & 5 & \\
& Otros & 10 & \multirow{2}{*}{33.9} \\
\cline { 2 - 3 } Torácica & Subtotal & 20 & \\
& T12-T13 & 16 & \\
& T13-L1 & 10 & \multirow{2}{*}{48.7} \\
\cline { 2 - 3 } & Otros & 13 & \\
\cline { 2 - 3 } & Subtotal & 39 & \multirow{2}{*}{100.0} \\
\hline Lumbar & L1-L2 & 10 & \\
& L2-L3 & 10 & \\
\hline Total & L7-S1 & 12 & \\
\hline
\end{tabular}


Cuadro 4. Distribución de los diagnósticos tomográficos por mayor ocurrencia según el espacio intervertebral afectado en 79 canes evaluados por tomografía axial computarizada

\begin{tabular}{|c|c|c|c|c|c|c|c|}
\hline \multirow{3}{*}{$\begin{array}{l}\text { Diagnósticos tomográficos } \\
\text { Hernia discal medial }\end{array}$} & \multirow{3}{*}{$\frac{\mathrm{n}}{26}$} & \multicolumn{6}{|c|}{ Espacios intervertebrales por regiones } \\
\hline & & \multicolumn{2}{|c|}{ Cervical } & \multicolumn{2}{|c|}{ Torácica } & \multicolumn{2}{|c|}{ Lumbar } \\
\hline & & $\mathrm{C} 4-\mathrm{C} 5$ & (4) & T13-L1 & (6) & L2-L3 & $(5)$ \\
\hline $\begin{array}{l}\text { Calcificación laminar del } \\
\text { saco dural }\end{array}$ & 9 & $\mathrm{C} 2-\mathrm{C} 3$ & (1) & $\mathrm{T} 12-\mathrm{T} 13$ & (2) & L4-L5 & (2) \\
\hline $\begin{array}{l}\text { Hernia discal paramedial } \\
\text { izquierda }\end{array}$ & 8 & $\mathrm{C} 5-\mathrm{C} 6$ & (2) & $\begin{array}{l}\mathrm{T} 11-\mathrm{T} 12 \\
\mathrm{~T} 12-\mathrm{T} 13\end{array}$ & $\begin{array}{l}(2) \\
(2)\end{array}$ & L4-L5 & (1) \\
\hline Protrusión discal & 6 & $\mathrm{C} 5-\mathrm{C} 6$ & (1) & $\mathrm{T} 11-\mathrm{T} 12$ & (1) & L7-S1 & (3) \\
\hline $\begin{array}{l}\text { Hernia discal paramedial } \\
\text { derecha }\end{array}$ & 5 & $\mathrm{C} 6-\mathrm{C} 7$ & (1) & $\mathrm{T} 12-\mathrm{T} 13$ & $(2)$ & L3-L4 & (1) \\
\hline $\begin{array}{l}\text { Hernia discal medial y } \\
\text { paramedial izquierda }\end{array}$ & 4 & $\mathrm{C} 6-\mathrm{C} 7$ & (1) & T12-T13 & $(2)$ & L7-S1 & (1) \\
\hline $\operatorname{Otros}^{1}$ & 21 & & 10 & & 23 & & 43 \\
\hline Total & 79 & & (20) & & (39) & & $(56)$ \\
\hline
\end{tabular}

1 Neoplasia vertebral, luxofractura, fusión intervertebral, estenosis foraminal bilateral, absceso vertebral. fractura de cuerpo vertebral, espondiloartrosis, enfisema medular, sindesmofitos, estenosis foraminal derecha, calcificación del ligamento amarillo, calcificación del ligamento longitudinal anterior, calcificación del ligamento longitudinal posterior, calcificación laminar del cordón medular, hernia discal medial y paramedial derecha, hernia discal paramedial izquierda y derecha

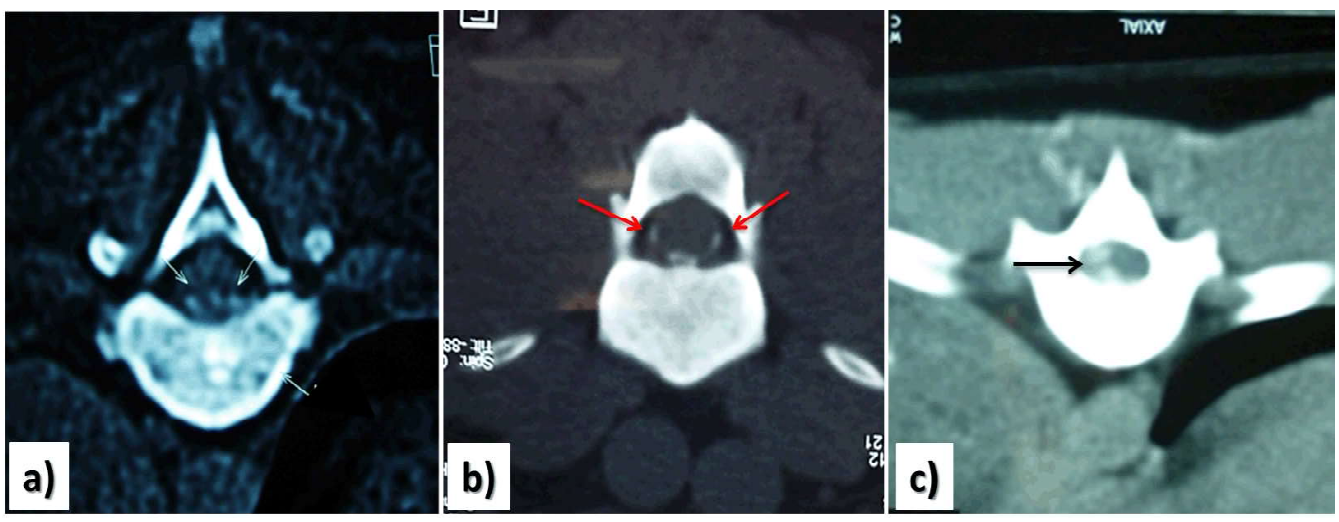

Figura 1. Tomografías transversas de columna vertebral a nivel de la región toraco lumbar: a) Hernia discal medial T12-T13 (flechas blancas) que produce estenosis del 40\% del canal medular. b) Calcificación lineal del saco dural al nivel de las vértebras T13-L1, asociado al 30-50\% del cordón medular (flechas rojas). c) Hernia discal para medial T12-T13 (flecha negra), que ocupa la mitad izquierda del canal medular produciendo compresión del cordón medular y se asocia a estenosis de los agujeros foraminales izquierdos 
Se obtuvieron 22 diagnósticos tomográficos dentro de los 79 casos. Los más frecuentes fueron la hernia discal medial (32.8\%, 26/79), calcificación laminar del saco dural $(11.4 \%, 9 / 79)$, hernia discal paramedial izquierda $(10.1 \%, 8 / 79)$ y protrusión discal $(7.5 \%, 6 / 79)$ (Cuadro 2).

De los 79 casos de lesiones en columna vertebral, el $69.6 \%$ presentó una lesión, el $22.8 \%$ presentó dos lesiones, el $2.5 \%$ tres presentó lesiones y el $5.1 \%$ presentó más de tres lesiones, lo cual indica un total de 115 lesiones diagnosticadas por tomografía computarizada.

Con respecto a las lesiones diagnosticadas por regiones de la columna vertebral, la región lumbar presentó la mayor frecuencia con el 48.7\% (56/115), donde los espacios intervertebrales más afectados fueron L1-L2, L2-L3 y L7-S1, la región torácica tuvo una de presentación del 33.9\% (39/115), donde los espacios intervertebrales más afectados fueron T12-T13 y T3-L1, y la región cervical fue del orden de $17.4 \%$ (20/115), donde los espacios intervertebrales más afectados fueron $\mathrm{C} 4-\mathrm{C} 5$ y C6-C7 (Cuadro 3).

La relación entre los diagnósticos tomográficos y los espacios intervertebrales por región de columna más afectados se presentan en el Cuadro 4. Cabe resaltar la mayor presentación de lesiones en espacio intervertebral T12-T13, con predominio de discopatías lateralizadas a izquierda y procesos degenerativos como la calcificación laminar del saco dural.

\section{Discusión}

El mayor número de lesiones diagnosticadas por TC correspondieron a canes machos, mayores de 4 años, afectados principalmente por discopatías. Para autores como Itoh et al. (2008) y Kazakos et al. (2012), el sexo no es determinante en la presentación de las enfermedades espinales en general; sin embargo, hay un significativo factor de riesgo en los machos para las discopatías, probablemente ligado a su mayor peso o a un supuesto efecto protector de los estrógenos frente a la degeneración del disco intervertebral (Chrisman et al., 2003; Fidalgo et al., 2003). Coates (2000) señala que los machos y hembras castradas tienen un alto riesgo de presentar hernias discales en comparación con hembras no castradas.

Las razas de tamaño mediano y condrodistróficas como el Beagle y el Shih Tzu fueron las más frecuentes con lesiones vertebrales y correspondieron a diagnósticos de hernia discal medial y paramedial, así como a calcificación del saco dural. Esto coincide con lo descrito en la literatura que indica que las razas condrodistróficas o con tendencia a la condrodistrofia son los canes más afectados por discopatías y alteraciones degenerativas de la columna (Itoh et al., 2008; Hecht et al., 2009). Cabe resaltar que el Dachshund no tuvo una alta frecuencia de lesiones, pese a ser históricamente la raza condrodistrófica más afectada por enfermedad del disco intervertebral con $45-65 \%$ de canes afectados (Coates, 2000). Este hallazgo podría deberse al bajo número de animales de esta raza en Lima en comparación con la población de otras razas.

Las discopatías intervertebrales fueron los diagnósticos tomográficos más frecuentes. Dentro de estas, la hernia discal medial fue la de mayor presentación (32.8\%), probablemente debido a la particular estructura anatómica del disco intervertebral. El anillo fibroso es más grueso en ventral y lateral que en dorsal, el núcleo pulposo tiene una localización excéntrica orientada hacia dorsal y el ligamento longitudinal dorsal es más frágil en comparación al ligamento longitudinal ventral, todo lo cual favorece la presentación dorsal o paramedial de las hernias (Smolders et al., 2013).

La calcificación laminar del saco dural (CSD) fue el segundo diagnóstico tomográfico de mayor presentación. Es una 
lesión con características degenerativas, presentándose en canes mayores de 8 años, mayormente en las razas Dachshund y Shih Tzu. La CSD se genera por el depósito de calcio a nivel del saco dural, pudiendo generar estenosis y compresión de la médula espinal que requiera una intervención quirúrgica para tratarla (Shiraishi et al., 2004). Las manifestaciones clínicas van desde el dolor en la región medular afectada hasta déficit neurológico. En el humano se han reportado lesiones de diversas etiologías como en columna cervical asociado a raquitismo hipofosfatémico ligado al cromosoma $\mathrm{X}$ (Najefi et al., 2017), estenosis espinal torácico por mielopatía torácica en un paciente con osteodistrofia renal (Wagle et al., 1993) y la calcificación dural en una persona con aracnoiditis asociado a síndrome de cauda equina (Bilgen et al., 1999). Si bien no existen a la fecha reportes en animales de compañía, se podría relacionar algunas de estas condiciones degenerativas o inflamatorias como causas probables de la CSD.

La hernia paramedial izquierda fue la tercera lesión diagnosticada con mayor frecuencia y conjuntamente con la hernia medial y paramedial derecha constituyeron más del $50 \%$ de los diagnósticos por TC del estudio. Este resultado coincide con lo establecido por Besalti et al. (2005), quienes encontraron la mayor frecuencia de extrusiones del núcleo pulposo a izquierda. El ligamento longitudinal dorsal esta dispuesto en la línea media en el canal medular, por lo que al extruirse el núcleo pulposo puede lateralizarse a izquierda $o$ derecha según el movimiento del paciente o la velocidad de la extrusión (Downes et al., 2009; Lim et al., 2010). Sin embargo, es importante mencionar que no hay estudios con referencias anatómicas que sustenten la mayor presentación de compresiones hacia algún lado en particular.

Las regiones de la columna vertebral con mayor número de lesiones fueron la lumbar y torácica. Estas lesiones se identificaron principalmente entre los espacios intervertebrales
T10 y L3 y correspondieron mayormente a discopatías. Esta relación puede ser explicada por la elevada movilidad de la columna a este nivel y la disminución en grosor del ligamento intercapital. Este ligamento está localizado en posición dorsal de los discos intervertebrales torácicos y une las cabezas de las costillas en la región que articulan con su vértebra correspondiente. Esta posición anatómica confiere un refuerzo natural, ayudando a prevenir lesiones en la médula por la extrusión o protrusión del disco entre las vértebras torácicas craneales a T10. Esta protección no es eficaz en la región toraco lumbar y explica por qué hay más frecuencia de hernias en esta región de la columna (De Lahunta y Glass, 2009).

Un factor adicional para la presentación de lesiones por discopatías a nivel toracolumbar es la estrechez anatómica de su canal medular al compararlo con la región cervical. En ese sentido, Arias et al. (2007) mencionan que cerca del $85 \%$ de los canes con discopatías presentan la lesión a nivel de la región toracolumbar y el 15\% en la región cervical. Esta condición anatómica también explica la diferencia en la presentación de los signos neurológicos, los cuales son más agudos y severos en la región toracolumbar y suelen ser más crónicos en la región cervical.

El espacio intervertebral más afectado fue T12-T13 con el 14\% (16/115) de los casos. Los diagnósticos tomográficos para este espacio incluyeron calcificación del saco dural, hernias paramediales derecha e izquierda, así como la asociación de hernia medial y paramedial izquierda. Este resultado coincide con lo encontrado por Maceiras (2014) quien en un estudio quirúrgico de casos por hernias discales encontró que el espacio intervertebral T12-T13 se presentó en un $32.8 \%$. Otros estudios refuerzan estos hallazgos reportándose hasta el $75 \%$ de ocurrencia para dicho espacio intervertebral (Simpson, 1992; Olby et al., 2003). 
En el presente trabajo, los diagnósticos tomográficos compatibles con neoplasias y luxofracturas de columna vertebral representaron el $2.5 \%$ con dos pacientes por cada diagnóstico, mientras que las neoplasias vertebrales se presentaron en canes de tamaño mediano de 8 y 16 años. Lorenz et al. (2004) mencionan que la médula espinal no es un sitio común para el desarrollo de neoplasias, aunque diversos estudios indican que los perros de gran talla parecen tener un riesgo mayor (Nelson y Couto, 2010; De Lahunta y Glass, 2009). Con relación a las fracturas vertebrales cabe destacar que la baja casuística obtenida puede responder a la poca utilización de la herramienta tomográfica para corroborar el diagnóstico radiográfico inicial. El traumatismo medular agudo causado por fracturas o luxo-fracturas representa una parte importante de la casuística en animales de compañía en muchos países de Latinoamérica como Chile, Argentina y Brasil (Bulcão et al., 2012), siendo frecuentemente asignada en canes como la segunda causa de afección medular después de las hernias discales (Shores, 1992; Bagley, 2000).

\section{Conclusiones}

- Las lesiones en columna vertebral más frecuentes en canes, diagnosticadas por tomografía computarizada, fueron: hernia discal medial (32.8\%), calcificación laminar del saco dural (11.4\%) y hernia discal paramedial izquierda (10.1\%).

- La región lumbar presentó la mayor frecuencia de lesiones $(48.7 \%)$.

- Las razas de tamaño mediano y condrodistroficas, como Beagle y Shih Tzu, fueron las más afectadas con diagnósticos de hernia discales y calcificación laminar del saco dural.

- El espacio intervertebral con mayor presentación de lesiones fue T12-13 con diagnósticos como calcificación laminar del saco dural y hernia discal medial y paramedial.

\section{Literatura Citada}

1. Arias MVB, Severo MS, Tudury EA. 2007. Trauma medular em cães e gatos: revisão da fisiopatologia e do tratamiento médico. Semina Ciências Agrárias 28: 115-134.

2. Axlund TW, Hudson JA. 2003. Computed tomography of the normal lumbosacral intervertebral disc in 22 dogs. Vet Radiol Ultrasound 44: 630-634. doi: 10.1111/j.1740-8261.2003.tb00521.x

3. Bagley RS. 2000. Spinal fracture or luxation. Vet Clin N Am-Small 30: 133 153. doi: 10.1016/S0195-5616(00)50006-0

4. Bali MS, Lang J, Jaggy A, Spreng D, Doherr MG, Forterre F. 2009. Comparative study of vertebral fractures and luxations in dogs and cats. Vet Comp Orthop Traumatol 22: 47-53.

5. Besalti O, Ozak A, Pekcan Z, Tong S, Eminaga S, Tacal T. 2005. The role of extruded disk material in thoracolumbar intervertebral disk disease: a retrospective study in 40 dogs. Can Vet J 46: 814-820.

6. Bilgen IG, Yunten N, Ustun EE, Oksel F, Gumusdis G. 1999. Adhesive arachnoiditis causing cauda equina syndrome in ankylosing spondylitis: CT and MRI demonstration of dural calcification and a dorsal dural diverticulum. Neuroradiology 41: 508-511.

7. Bray J, Burbigde H. 1998. The canine intervertebral disc. Part two: degenerative changes - noncondrodys-trophoid versus condrodystrophoid disks. J Am Hosp Assoc 34: 135-144. doi: 10.5326/ 15473317-34-2-135

8. Bulcão A, Da Paz Junior CJ, Silva RO. 2012. Estudo retrospectivo da ocorrência de fratura de coluna em cães e gatos atendidos no laboratório de radiologia da clínica veterinária $\mathrm{da}$ ESBAM, Manaus, AM. Amazon Sci 1(1): 25-27.

9. Chrisman C, Mariani C, Platt S, Clemmons R. 2003. Manual de neurología práctica. España: Multimédica. 368 p. 
10. Coates J. 2004. Paraparesis. In: Platt $\mathrm{S}$, Olby, N (eds). BSAVA Manual of canine and feline neurology. $3^{\text {th }}$ ed. England: British Small Animal Veterinary Asociation. p 237-264.

11. Coates JR. 2000. Intervertebral disk disease. In: Thomas WB (ed). The veterinary clinics of North America: small animal practice - common neurologic problems. Philadelphia: Saunders. p 77-104.

12. De Lahunta A, Glass E. 2009. Small animal spinal cord disease. In: De Lahunta A, Glass E (eds). Veterinary neuroanatomy and clinical neurology. $3^{\text {th }}$ ed. St. Louis, Missouri, USA: Saunders Elsevier. p 243-284.

13. Dennis R. 2003. Advanced imaging: indications for $\mathrm{CT}$ and MRI in veterinary patients. In Practice 25: 243-255. doi: 10.1136/inpract.25.5.243

14. Downes C, Gemmill T, Gibbons S, McKee W. 2009. Hemilaminectomy and vertebral stabilization for the treatment of thoracolumbar disc protrusion in 28 dogs. J Small Anim Pract 50: 525-535. doi: 10.1111/j.1748-5827.2009.00808.x

15. Fidalgo Álvarez LE, Rejas López J, Ruiz de Gopegui R, Ramos Antón JJ. 2003. Patología médica veterinaria. España: Universidad de León. $616 \mathrm{p}$.

16. Hecht S, Thomas W, Marioni-Henry $K$, Echandi R, Matthews A, Adams W. 2009. Myelography vs computed tomography in the evaluation of acute thoracolumbar intervertebral disk extrusion in chondrodystrophic dogs. Vet Radiol Ultrasound 50: 353-359. doi: 10.1111/j.1740-8261.2009.01549.x

17. Itoh H, Hara Y, Yoshimi N, Harada Y, Nezu Y, Yogo T, Ochi H, et al. 2008. A retrospective study of intervertebral disc herniation in dogs in Japan: $297 \mathrm{Ca}-$ ses. J Vet Anim Sci 70: 701-706.

18. Kazakos G, Polizopoulou Z, Patsikas M, Tsimopoulos G, Roubies N, Dessiris A. 2005. Duration and severity of clinical signs as prognostic indicators in $30 \mathrm{dogs}$ with thoracolumbar disk disease after surgical decompression. J Vet Med A Physiol Pathol Clin Med 52: 147-152. doi: 10.1111/j.1439-0442.2005.00698.x

19. Lecouteur A, Grandy J. 2002. Enfermedades de la médula espinal. En: Ettinger SJ (ed). Tratado de medicina interna veterinaria. Enfermedades del perro y el gato. $5^{\text {a }}$ ed. Argentina: InterMédica. p 677-732.

20. Lim C, Kweon OK, Choi MC, Choi J, Yoon J. 2010. Computed tomographic characteristics of acute thoracolumbar intervertebral disc disease in dogs. J Vet Sci 11: 73-79. doi: 10.4142/jvs.2010.11.1.73

21. Lorenz, DM, Coates J, Kent M. 2011. Handbook of veterinary neurology. $5^{\text {th }} \mathrm{ed}$. St Louis, Misouri: Saunders. 560 p.

22. Maceiras M. 2014. Estudio descriptivo de casos de perros con hernia discal toracolumbar tipo I sometidos a hemilaminectomía entre los años 20012010. Tesis de Médico Veterinario. Santiago de Chile: Univ. de Chile. 35 p.

23. McKee M. 2000. Intervertebral disc disease in the dog. 1. Pathofisiology and diagnosis. In Practice 22: 355-369. doi: 10.1136/inpract.22.7.355

24. Najefi AA, Beder DB, Sabah SA, Rezajooi K. 2017. Cervical dural calcification and cervical myelopathy in X-linked hypophosphataemic rickets: a case report and review of the literature. Br J Neurosurg 25: 1-2. doi: 10.1080/ 02688697.2017.1319908

25. Nelson RW, Couto CGL. 2010. Medicina interna de pequeños animales. $4^{\mathrm{a}} \mathrm{ed}$. España: Elsiever. 1505 p.

26. Olby NJ, Muñana KR, Sharp NJ, Thrall DE. 2000. The computed tomographic appearance of acute thoracolumbar intervertebral disc herniations in dogs. Vet Radiol Ultrasound 41: 396-402. doi: 10.1111/j.1740-8261.2000.tb01860.x

27. Olby N, Levine J, Harris T, Muñana K, Skeen T, Sharp N. 2003. Longterm functional outcome of dogs with severe injuries of the thoracolumbar spinal cord: 
87 cases (1996-2001). J Am Vet Med Assoc 222: 762-769. doi: 10.2460/ javma.2003.222.762

28. Oppenheimer A. 1942. Calcification and ossification of the vertebral ligament (spondylitis ossificans ligamentosa): roentgen study of pathogenesis and clinical significance. Radiology 38: 160173. doi: $10.1148 / 38.2 .160$

29. Pellegrino F. 2014. Las claves del diagnóstico neurológico para el veterinario clínico. Argentina: Inter-Médica. 456 p.

30. Shiraishi T, Ikegami T, Okubo Y, Yato Y, Honda M. 2004. Cervical peridural calcification in patients undergoing longterm hemodialysis. Report of two cases. J Neurosurg_Spine 100: 284-286.

31. Shores A. 1992. Fractures and luxations of the vertebral column. Vet Clin NAmSmall 22: 171-180. doi: 10.1016/S01955616(92)50012-2
32. Simpson S. 1992. Intervertebral disc disease. Vet Clin NAm-Small 22: 889-897.

33. Smolders L, Kingma L, Bergknut N, van der Veen A, Dhert W, Hazenwinkel H, van Dieen J, Meij B. 2013. Biomechanical assessment of the effects of decompressive surgery in nonchondrodystrophic and chondrodystrophic canine multisegmented lumbar spines. Eur Spine J 21: 1692-1699. doi: 10.1007/s00586-012-2285-0

34. Wagle VG, Rossi AJ, Roberts MP, Goldman R, Ziter F, Clark WE. 1993. Thoracic spinal stenosis associated with renal osteodystrophy. Diagnosis based on magnetic resonance imaging and computed tomography. Spine 18: 1373-1375.

35. Widmer W, Thrall D.2013. The canine and feline vertebrae. In: Thrall D (eds). Textbook of veterinary diagnostic radiology. $6^{\text {th }}$ ed. St. Louis, Missouri: Elsevier Saunders. P 172-191. 